\title{
Humanização como forma de superação da violência obstétrica: papel do enfermeiro
}

\author{
Humanization as a form of obstetric violence overcoming: role of the nurse
}

Humanización como forma de superación de la violencia obstétrica: papel del enfermero

Taciany Alves Batista Lemos ${ }^{1 *}$, Bruna de Abreu Sepulvedra ${ }^{2}$, Thaysa Batista Vieira de Rezende ${ }^{3}$, Luciana de Carvalho Coelho Chagas $^{4}$, Monique Caroline da Costa Silva ${ }^{5}$, Ana Raquel Xavier Meneses $^{6}$, Laurice Alves dos Santos ${ }^{7}$.

\begin{abstract}
RESUMO
Objetivo: Demonstrar o papel do enfermeiro nesse processo de humanização da assistência. Métodos: Os dados foram obtidos através da seleção de artigos relacionados ao tema nas bases de dados eletrônico Lilacs e Scielo, BVS, a amostra final foi composta por 08 artigos publicados entre os anos 2011 a 2018. Que foram agrupados em três grandes temas centrais. Resultados: Após breve recuperação histórica do tema, mapeiam-se o papel do enfermeiro obstetra no processo de humanização do parto. Caracterizar da violência obstétrica no Brasil. Discutese a complexa causalidade destas formas de violência, incluindo o papel do enfermeiro e as implicações na morbimortalidade materna. Conclusão: Portanto, faz-se necessário mudanças no modelo da assistência obstétrica. Os resultados desta revisão expressam necessidade de mudanças assistenciais vigentes, com o intuito de reduzir procedimentos desnecessários e as violações dos direitos das mulheres.
\end{abstract}

Palavras-chave: Humanização do Parto, Violência Obstétrica, Enfermagem.

\begin{abstract}
Objective: To analyze in the literature, the Brazilian panorama situations of obstetric violence in the prepartum, delivery and postpartum. And as a specific objective: Demonstrate the role of the nurse in this process of humanization of care. Methods: The data were obtained through the selection of articles related to the theme in the electronic databases Lilacs and Scielo, BVS, the final sample was composed by 08 articles published between the years 2011 to 2018. They were grouped into three main central themes. Results: After a brief historical recovery of the theme, the role of the obstetrician nurse in the process of humanization of childbirth is mapped. Characterization of obstetric violence in Brazil. We discuss the complex causality of these forms of violence, including the role of nurses and the implications for maternal morbidity and mortality. Conclusion: Therefore, it is necessary to change the model of obstetric care. The results of this review express the need for current assistance changes in order to reduce unnecessary procedures and violations of women's rights.
\end{abstract}

Key words: Humanization of Childbirth, Obstetric Violence, Nursing.

\section{RESUMEN}

Objetivo: Analizar en la literatura, el panorama brasileño situaciones de violencia obstétrica en el pre-parto, parto y posparto. Y como objetivo específico: Demostrar el papel del enfermero en ese proceso de humanización de la asistencia. Métodos: Los datos fueron obtenidos a través de la selección de artículos relacionados al tema en las bases de datos electrónicos Lilacs y Scielo, BVS, la muestra final fue compuesta por 8 artículos publicados entre los años 2011 a 2018. Que fueron agrupados en tres grandes temas centrales. Resultados: Tras una breve

${ }^{1}$ Enfermeira, Mestranda em Biotecnologia pela Faculdade Integral Diferencial - FACIDMYDEN, Teresina, Piauí. *E-mail:tacianyablemos@hotmail.com

${ }^{2}$ Enfermeira pela Universidade Federal do Piauí (UFPI).

${ }^{3}$ Enfermeira, pós-graduada em MBA em auditória em Serviços de saúde pela UNIPÓs. Teresina-PI.

${ }^{4}$ Enfermeira, Pós-Graduada em Unidade de Terapia intensiva. Teresina-PI.

${ }^{5}$ Enfermeira, Especialista em Enfermagem Obstétrica pela Diferencial-WYDEN-FACID Teresina-PI.

${ }^{6}$ Enfermeira, esp. em Qualidade e Segurança no cuidado ao paciente pelo Sírio Libanês. Santa Catarina SC. ${ }^{7}$ Enfermeira, Especialista em Docência do Ensino Superior pela Faculdade Aldemar Rosado (FAR), Teresina-PI. 
recuperación histórica del tema, se asignan el papel del enfermero obstetra en el proceso de humanización del parto. Caracterizar de la violencia obstétrica en Brasil. Se discute la compleja causalidad de estas formas de violencia, incluyendo el papel del enfermero y las implicaciones en la morbimortalidad materna. Conclusión: Por lo tanto, se hace necesario cambios en el modelo de la asistencia obstétrica. Los resultados de esta revisión expresan la necesidad de cambios asistenciales vigentes, con el fin de reducir procedimientos innecesarios y las violaciones de los derechos de las mujeres.

Palabras Clave: Humanización del Parto, Violencia Obstétrica, Enfermería.

\section{INTRODUÇÃO}

Ao decorrer de muitos anos, as mulheres vêm sendo vítimas de diversas formas de violências. O conceito de humanização da assistência ao parto inclui vários aspectos que de acordo com a Organização Mundial da Saúde (OMS, 2014), que pode ser entendida como um conjunto de condutas, atitudes e posturas, que vai desde $\mathrm{o}$ acolhimento da paciente até o momento pós-parto.

Diante disto, entende-se que violência obstétrica é um tipo de violência contra a mulher, em que ocorre graus significativos de pressão psicológica chegando à dor e sofrimento físico que poderiam ser evitadas. Destaca-se nesta perspectiva que o descaso e desrespeito com a gestante no pré parto, parto, pós parto e puerpério ocorre tanto no setor público quanto no setor privado de saúde, tomando lugar de destaque na imprensa e nas mídias sociais, por meio de relatos e depoimentos de mulheres que vivenciaram essas experiências (D'OLIVEIRA, AFPL DINIZ, CSG SCHRAIBER LB, et al., 2012).

Ainda, nesse contexto, a violência obstétrica é um fenômeno que vem acontecendo há algumas décadas, e pode ser entendida como uma realidade que pode ocorrer na gestação, no parto, no pós-parto ou em situações de abortamento. De acordo com García D et al., (2013) um fator predominante entre as gestantes é a desinformação e o receio em perguntar sobre os processos que serão realizados na evolução do trabalho de parto.

Situações como essas descritas acima podem levá-las a se conformarem com a exploração de seus corpos por diferentes profissionais, aceitando sem reclamar diversas situações incomodas que poderiam ser evitadas. Segundo a OMS (2014), gestantes no mundo todo tem sofrido abusos, desrespeitos (gritos e ofensas da equipe de saúde), episiotomia (corte no períneo) sem o consentimento, proibição de acompanhante na hora do parto e não permanência do filho junto à mãe logo após o nascimento, negligência e maus-tratos durante o parto nas instituições de saúde entre outras.

A OMS (2014) aponta a necessidade de estudos aprofundados a respeito desta temática com o intuito de compreender, definir, medir os graus de comprometimento psicológico a fim de melhorar as práticas de assistência, respeitando os direitos das mulheres durante o parto, assim como elaborar e disseminar informações a respeito da prevenção e eliminações de tais condutas.

No Brasil, vive-se hoje, o que se pode denominar de paradoxo perinatal: ao mesmo tempo em que há melhoras significativas na ampliação do acesso das mulheres aos serviços de saúde e à disponibilização de tecnologias para diagnóstico, há uma intensa medicalização do parto e do nascimento, com a manutenção de taxas elevadas de morbimortalidade materna e perinatal. Isto sinaliza uma baixa qualidade da atenção ao pré-natal e ao parto. A taxa de $53,7 \%$ de cesarianas no Brasil é um dos exemplos mais enfáticos desta realidade (LANSKY S, et. al. 2014). Além disso, nesse contexto Lansky S, et al., (2014), aponta que reduzir o número de cesarianas e de outras intervenções desnecessárias na assistência ao parto é uma tarefa complexa e que está alem do espectro da saúde. Envolvendo questões de igualdade de gênero, acesso a renda e a educação.

De acordo com a Portaria n. 1.459/2011 do Ministério da Saúde, (2014);

"Considerando a prevalência de partos hospitalares e o aumento do número de cesáreas registradas no Brasil, assim como o atual cenário de práticas e intervenções descrito, verifica-se a importância de analisar a assistência à gestação e ao parto, compreendendo todo o período, desde as consultas pré-natais até o pós-parto". 
A ocorrência de violência obstétrica é uma realidade que devem ser combatidas, através da qualidade da assistência prestada, e a participação da mulher no processo do cuidado, levando em consideração as informações repassadas pela gestante e seu consentimento em relação a sua situação real de saúde, a explicação real dos riscos e benefícios de cada procedimento a ser realizado, e a garantia do direto de ter um acompanhante nesse processo tendo com foco uma política de humanização do atendimento (MINISTÉRIO DA SAÚDE, 2014).

Sendo assim, diante do exposto e considerando a relevância do tema têm-se como objetivo geral do estudo: Analisar na literatura, o panorama brasileiro situações de violência obstétrica no pré parto, parto e pós-parto, incluindo os aspectos geradores deste tipo de violência. E como objetivo específico: Demonstrar nas evidências científicas acerca do papel do enfermeiro nesse processo de humanização da assistência, compilando as principais produções da área que evidenciam o processo de humanização. Analisando também o atual panorama brasileiro das práticas assistenciais a gestante, parturiente, e no pós-parto. Com o intuito de possibilitar a reflexão e explorar outras perspectivas neste contexto, melhorando as práticas assistenciais.

\section{MÉTODOS}

Trata-se de uma revisão de narrativa, na qual possibilita a síntese de pesquisas acerca da violência obstétrica, as principais situações recorrentes de violência no momento do pré-parto, parto e pós-parto a falta de garantia dos direitos da parturiente, e como o processo de humanização modifica esse processo.

A amostra final foi composta por 08 artigos. Para tanto estabeleceu-se os seguintes critérios de inclusão: Publicações em texto completo acessível que estivessem disponíveis online gratuitamente, no ano de publicação de 2011 a 2018, na língua portuguesa, foi utilizado o vocabulário estruturado de Descritores em Ciências da Saúde (DeCS), através dos seguintes descritores e operadores booleanos and: AND "Humanização do Parto" AND "Violência obstétrica" AND "Enfermagem", Foram encontrados 71 artigos, dos quais foram pré-selecionados 21 e posteriormente selecionados 8 (Descritos no quadro 1) que estavam de acordo com o tema proposto no trabalho, sendo realizada uma seleção dos artigos previamente, com a finalidade de confirmar a temática proposta e incluir somente estudos potencialmente relevantes. Que foram agrupados em três grandes temas centrais.

Quadro 1 - Síntese dos artigos selecionados na revisão narrativa da literatura brasileira. Teresina- PI, 2018.

\begin{tabular}{|c|c|c|c|}
\hline Autores & $\begin{array}{c}\text { Ano de } \\
\text { publicacão }\end{array}$ & Título do Trabalho & Periódico \\
\hline CASATE JC, CORRÊA AK. & 2017 & $\begin{array}{l}\text { Humanização do atendimento em } \\
\text { saúde: conhecimento veiculado na } \\
\text { literatura brasileira de enfermagem }\end{array}$ & $\begin{array}{ll}\text { Revista } & \text { Latino-Am } \\
\text { Enfermagem } & \end{array}$ \\
\hline CAUS ECM, et al. & 2017 & $\begin{array}{l}\text { O processo de parir assistido pela } \\
\text { enfermeira obstétrica no contexto } \\
\text { hospitalar: significados para as } \\
\text { parturientes }\end{array}$ & Revista Esc Anna Nery \\
\hline DINIZ CSG. & 2015 & $\begin{array}{l}\text { Gênero, saúde materna e o paradoxo } \\
\text { perinatal }\end{array}$ & $\begin{array}{lr}\text { Revista Brasileira de } \\
\text { Crescimento e } \\
\text { Desenvolvimento Humano }\end{array}$ \\
\hline GUERREIRO EM et al. & 2012 & $\begin{array}{l}\text { O cuidado pré-natal na atenção básica } \\
\text { de saúde sob o olhar de gestantes e } \\
\text { enfermeiros }\end{array}$ & Revista Min. Enferm \\
\hline LEAL MC, et al. & 2014 & $\begin{array}{l}\text { Intervenções obstétricas durante } 0 \\
\text { trabalho de parto e parto em mulheres } \\
\text { brasileiras de risco habitual. }\end{array}$ & Revista Cad. saúde pública \\
\hline NARCHI NZ, et al. & 2017 & $\begin{array}{l}\text { O papel das obstetrizes e enfermeiras } \\
\text { obstetras na promoção da maternidade } \\
\text { segura no Brasil. }\end{array}$ & $\begin{array}{lll}\text { Revista } & \text { Ciênc } & \text { Saúde } \\
\text { Coletiva. } & & \\
\end{array}$ \\
\hline SILVA AA, et al. & 2015 & $\begin{array}{l}\text { Violência Obstétrica: Perspectiva da } \\
\text { Enfermagem }\end{array}$ & $\begin{array}{l}\text { Revista Rede de Cuidados } \\
\text { em Saúde. }\end{array}$ \\
\hline WOLFF LR, WALDOW VR & 2018 & $\begin{array}{l}\text { Violência consentida: mulheres em } \\
\text { trabalho de parto e parto }\end{array}$ & $\begin{array}{lll}\text { Revista } & \text { Saúde } & \text { e } \\
\text { Sociedade. } & & \\
\end{array}$ \\
\hline
\end{tabular}

Fonte: Pesquisa direta, 2018. 
Com levantamento bibliográfico realizado através da seleção de artigos relacionados ao tema nas bases de dados Scientific Electronic Library Online (SCIELO), BDENF (Base de dados em Enfermagem), Lilacs, Biblioteca Virtual de Saúde (BVS).

\section{RESULTADOS}

\section{Abordagens históricas sobre o parto no brasil}

No final do século $X X$ a assistência ao parto era uma prática desenvolvidas pelas mulheres, realizadas nas casas das famílias da gestante, com o acompanhamento das parteiras ou comadres, que eram pessoas de confiança da gestante, pessoas que eram consideradas com grande saber nos mecanismos de reprodução e que envolviam dimensões biológicas, e psicológicas ao ato de nascer. O parto e o nascimento, que eram vistos como um evento fisiológico e feminino, e o ato de cuidados com a mulher durante o parto, sempre esteve ligado ao fazer feminino desde os tempos imemoriais, e consolidou este espaço como local de poder feminino (WOLFF LR, WALDOW VR, 2018).

O trabalho das parteiras dotadas de muito conhecimento, tendo em vista sua vasta experiência em gravidezes e partos envolve a prática de solidariedade feminina, sendo conduzido como um evento natural e fisiológico dentro das comunidades, as parteiras eram dotas de algumas características comuns como: conselheiras, curadoras e amigas das famílias que necessitam dos seus serviços além de possuir uma experiência corporal e emocional que garantia prestígio e competência para as parteiras e exercitavam suas alianças de gênero (LEAL MC, PEREIRA APE, DOMINGUES RMSM, et. al., 2014).

Conforme disposto por Brugemann OM (2015) A partir do sec. XVIII, a arte de partejar, historicamente constituída como saber-poder feminino, rico em saberes populares, é transformada em saber-poder masculino com a progressiva medicalização da sociedade. Surge a medicina científica e masculina, que passa a ser desenvolvida nas universidades de acesso exclusivo aos homens e dá continuidade ao processo de normalização do corpo feminino iniciado pela igreja no sec. XV.

Com a entrada dos médicos neste cenário, do parto, as parteiras passara a ocupar segundo plano e as mulheres passaram a atuar como coadjuvantes no trabalho de parto, é inegável, que o parto é um momento único, cheio de significados e inesquecível na vida da gestante, devendo ser singular e tendo como foco 0 protagonismo da mulher (LEAL MC, PEREIRA APE, DOMINGUES RMSM, et. al., 2014).

A prática da assistência ao parto no século XX, mesmo com a participação limitada das parteiras, passou a ser vista como um ato privativo médico, tendo como predominância o parto hospitalar, sobretudo após a segunda guerra mundial. A assistência foi se mecalizando e masculinizando, neste período tem início o desenvolvimento do modelo assistencial biomédico, no qual o paciente é visto como um sujeito passivo, sem autonomia, incapaz de tomar decisões nem opinar sobre o que the convêm (LEAL MC, PEREIRA APE, DOMINGUES RMSM, et al., 2014; WOLFF LR, WALDOW VR, 2018).

De acordo com Costa LHR, 2015, no contexto do parto, a mulher é esquecida e tomada como objeto, um sujeito passivo cuja opinião é insignificante, em que a subjetividade desconsiderada nesta relação. O serque-gera é sempre visto a partir do seu caráter reprodutivo e sua singularidade é negada, tendo seu corpo e sexualidade controlados pela medicina.

No Brasil, atualmente, intensificam-se vários movimentos contrários à medicalização da gravidez, liderados por mulheres e ativistas, ao mesmo tempo em que ocorre um paradoxo perinatal com melhoras significativas de ampliação do acesso de mulheres ao serviço de saúde de qualidade com o auxilio da tecnologia avançada para auxilio de diagnóstico, há em contrapartida uma intensa medicalização do parto e nascimento com aumento de taxas nos marcadores de morbimortalidade materna e perinatal (LANSKY S, et al., 2014).

Para Diniz CSG (2015), mesmo assim, a violência obstétrica esteve presente em iniciativas como as capacitações para o atendimento a mulheres vítimas de violência, como nos cursos promovidos a partir de 
1993, pelo Coletivo Feminista Sexualidade e Saúde e pelo Departamento de Medicina Preventiva da USP. A partir deste projeto, foi publicado um pequeno manual sobre o tema.

Por esta razão, deve-se combater a prática da violência obstétrica através da garantia dos direitos as gestantes, assistência de qualidade ao pré-natal, pré-parto, parto, puerpério e situações de abortamento, garantindo a humanização em todo ciclo gravídico puerperal. Esta assistência deve envolver vários aspectos que beneficiam as gestantes, como autonomia, compromisso, respeito, empatia, escuta ativa, englobando desta forma, a mulher como biopsicossocial, que passa por modificações psicológicas, físicas, espirituais, sociais, culturais, não se restringindo apenas a aspectos biológicos a gestantes (BRASIL, 2016).

\section{Papel do enfermeiro obstetra no processo de humanização do parto}

Em essência, humanizar o parto e o nascimento é um movimento de contracultura que busca resgatar a humanidade e a individualidade da mulher que pare, em oposição ao ideário do corpo máquina, da mulher como fábrica de bebês e da maternidade como linha de montagem (NUNES DC, SILVA LAM, 2012).

O conceito de humanização é amplo e polissêmico, que envolve conhecimentos, práticas e as atitudes que tem como objetivos promover partos e nascimentos saudáveis com os diretos garantidos em sua integralidade, garantindo o direito a privacidade, a autonomia e a mulher assumindo posição de protagonismo, ofertando procedimentos que tragam benéficos comprovados, evitando intervenções desnecessárias e que sejam capazes de prevenir e aumentar a morbimortalidade materna e fetal. O modelo de humanização do parto pressupõe que segurança não é sinônimo de intervenção e tecnologia. Ao contrário, na assistência ao parto, na maioria das vezes segurança e expectativa são uma e a mesma coisa, sendo o modelo tecnocrático muito mais ritualístico do que científico (MARTINELLI et al., 2014; NUNES DC, SILVA LAM, 2012, DINIZ CSG, 2015).

Cabe salientar, que o profissional enfermeiro exerce um papel fundamental neste processo de humanização através de um olhar holístico, e integral, minimizando técnicas invasivas episiorrafias indiscriminadas que podem levar a inúmeras complicações como: infecções, hematomas, extensão do períneo, edemas locais, dispaurenias, hemorragias, cicatrizes, além de sempre levar em consideração a autonomia, decisão sem interferência que possam interferir negativamente na qualidade de vida das mulheres (DINIZ CSG, 2015).

No Brasil, políticas de humanização estão em ascensão e cada vez mais sendo disseminada a população para conscientização das mulheres acerca dos seus direitos, algumas medias como a criação da politica de humanização do parto e nascimento (REHUMA) no ano de 1993, Programa de Humanização no pré-natal e nascimento (PHPN) nos anos 2000 que se tornaram marco da promoção dos direitos humanos das mulheres, destacando durante o trabalho de parto e no momento do parto. Sendo assim, Brasil, (2006); Nunes DC, (2012), destacam que uma assistência humanizada se refere à prestação de uma assistência que prioriza a qualidade do cuidado garantindo respeito aos direitos e sua individualidade, valorizando a autonomia, protagonismo e cor responsabilização dos sujeitos envolvidos no processo do cuidado. É uma forma de transformar o processo de desumanização que se tornou cada vez mais gradativa, em procedimentos que visam a suavizar as dores e temores de pacientes que necessitam de atendimento e cuidados.

Nesse contexto, os profissionais de saúde, destacando o enfermeiro, neste processo, é responsável pela assistência e acompanhamento ao pré-natal de baixo risco nas estratégias de saúde da família, devendo orientar as puérperas ao decorrer de todo processo gravídico através de ações individuais e grupais, de forma prestativa e conquistando a confiança, atenuando ansiedades, medos, receios, angustias, dúvidas, garantindo-Ihes empoderamento sobre direitos durante o trabalho de parto e parto, dentre outras ações que tem como principal enfoque diminuir as intervenções que caracterizam violência obstétrica nas UBS e seus prevendo potenciais riscos à saúde da mulher e do bebê promovendo humanização da assistência tanto em maternidades, como em casas de parto (GUERREIRO EM, et al 2012; SILVA AA. SOUZA TGG, MUNHOZ MA, et al., 2015). 
Infere-se que, o primeiro contato da gestante com o serviço de saúde se baseia em ações pautadas em uma assistência progressiva e integral, baseadas no acolhimento e atenção às demandas de saúde, contribuindo para um trabalho humanizado e qualificado. Compreendendo todos os esforços para evitar condutas intempestivas e agressivas para o binômio mãe-bebê. Esclarecer rotinas e procedimentos realizados de forma tranquila e passando confiança para mulher. Assim, conceituar a humanização do parto e nascimento, pode-se compreender como um conjunto de práticas e procedimentos que buscam um movimento para readequar o processo do parto pautado na individualidade e singularidade feminina, com uma menor medicalização e hospitalização, com uma visão global, valorizando o protagonismo feminino que permita um cuidado com oposição ao modelo tradicional, permitindo uma maior congruência de crenças, valores e cultura (CASATE JC, CORRÊA AK, 2017).

Destaca-se que o processo de humanização da assistência ao parto e nascimento implica em modificações da assistência por meio de condutas que garantam o respeito e de forma sensível e que pode ajudar a mulher na tomada de decisão, garantindo qualidade do cuidado e estreitando o elo entre o trinômio mulher-criança-família. Tal processo além de valorizar a mulher, envolve empatia, respeito a singularidade, e garantia de um ambiente seguro e acolhedor (CASATE JC, CORRÊA AK, 2017).

Assim, corrobora a necessidade de tornar cada vez mais humano este processo, entre os principais nortes da humanização ao parto estão: protagonismo da mulher e suas escolhas, conscientização e capacitação com ações educativas permanentes em saúde. Considerar o momento não como um evento médico, mas emocional, social, e espiritual e familiar. A formação profissional diferenciada em saúde com eixo transversal devendo enxergar o nascimento de forma natural, sem interferências desnecessárias (NARCHI NZ, CRUZ EF, GONÇALVES R, 2017; SOUSA AMM, SOUZA KV, REZENDE EM, et al., 2017; CAUS ECM, SANTOS EKA, NASSIF AA, et. al, 2017).

\section{CONSIDERAÇÕES FINAIS}

A pesquisa permitiu constatar que a violência obstétrica é frequente, no Brasil, e praticada por médicos e profissionais da enfermagem, em especial, na forma de negligência, violência verbal e violência física, exceto a violência sexual. No entanto, diversas estratégias esbarram no modelo biomédico de atenção práticas com intervenções abusivas, baixa valorização da gestante que deixa de ser protagonista deste processo, causando danos biopsicossociais. Desta forma, este estudo destaca a importância de minimizar até anular definitivamente práticas abusivas e prejudiciais, praticadas por profissionais com uso de estratégias que efetivem programas e políticas públicas já implementadas voltadas ao binômio (mão e filho). Portanto, fazemse necessárias mudanças no modelo da assistência obstétrica, na grade curricular durante a formação dos profissionais em todas as categorias de cursos superior em Obstetrícia, para diferenciação da formação médica tradicionalista, fato este que acontece anos.

\section{REFERÊNCIAS}

1. ANDRADE BP, AGGIO CM. Violência obstétrica: a dor que cala. Londrina, Anais do III Simpósio Gênero e Políticas Públicas, 2014. ISSN 2177-8248. Acesso em 29/06/2018 Disponível em: http://www.uel.br/eventos/gpp/pages/arquivos/GT3_Briena\%20Padilha\%20Andrade.pdf.

2. BRASIL. Ministério da Saúde. Atenção à saúde da gestante em APS. Organização de Maria Lucia Medeiros Lenz, Rui Flores. - Porto Alegre: Hospital Nossa Senhora da Conceição, 2016. p. 240 v: 30. Acesso em 29/06/2018 Disponível em: http://www2.ghc.com.br/gepnet/publicacoes/atencaosaudeda-gestante.pdf.

3. BRUGEMANN OM. Resgatando a história obstétrica para vislumbrar a melodia da humanização. In: ZAMPIERE, FM, OLIVEIRA ME, BRUGGMANN OMA, melodia da humanização: reflexões sobre o cuidado no processo de nascimento. Florianópolis (SC): Cidade Futura, 2015. p. 23-26. 2.

4. CASATE JC, CORRÊA AK, Humanização do atendimento em saúde: conhecimento veiculado na literatura brasileira de enfermagem. Rev Latino-Am Enfermagem [Internet]. 2005; [cited 2017 Mar 17]; 13(1):105- 11. Acesso em 25/06/2018. Disponível em: http://www.scielo.br/pdf/rlae/v13n1/v13n1a17.pdf. 
5. CAUS ECM, SANTOS EKA, NASSIF AA, et. al, O processo de parir assistido pela enfermeira obstétrica no contexto hospitalar: significados para as parturientes. Esc Anna Nery [Internet]. 2012 Mar; [cited 2017 Mar 16]; 16(1):34-40. Acesso em 28/06/2018. Disponível em: http://www.scielo.br/pdf/ean/ v16n1/v16n1a05.pdf.

6. COSTA LHR, Corpo, poder e o ato de partejar: reflexões à luz das relações de gênero. Rev. bras. enferm. 2015; 53(1):39-46. Acesso em 29/06/2018 Disponível em: http://www.scielo.br/pdf/reben/v53n1/v53n1a06.pdf.

7. D'OLIVEIRA AFPL, DINIZ CSG, SCHRAIBER LB, Violence against women in health care institutions: an emerging problem. Rev. Lancet, 2012 359(11), 16811685.

8. DINIZ CSG. Gênero, saúde materna e o paradoxo perinatal. Revista Brasileira de Crescimento e Desenvolvimento Humano, 2015. Vol 19(2), 313-326. Acesso em: Acesso em 29/06/2018 Disponível em: http://www5.usp.br/90463/revista-brasileira-de-crescimento-e-desenvolvimento-humano.

9. FIOCRUZ. Nascer no Brasil: pesquisa revela número excessivo de cesarianas. 2014. Acesso em 29/06/2018 Disponível em: https://portal.fiocruz.br/noticia/nascer-no-brasil-pesquisa-revela-numero-excessivo-de-cesarianas.

10. GARCÍA D, DÍAZ Z. ACOSTA M. El nacimiento em Cuba: análisis de la experiencia del parto medicalizado desde una perspectiva antropológica. Revista Cubana de Salud Pública, 2013. 39(4), 718-732. Acesso em 29/06/2018 Disponivel em: http://dx.doi.org/10.1590/S141381232012000700029.

11. GUERREIRO EM. et al. O cuidado pré-natal na atenção básica de saúde sob o olhar de gestantes e enfermeiros. Rev. Min. Enferm. 2012. 16(3): 315-323, jul./set. Acesso em 29/06/2018 Disponível em: DOI: http://www.dx.doi.org/S1415-27622012000300002.

12. LANSKY S, FRICHE AAL, SILVA AAM, et al. Pesquisa nascer no brasil: perfil da mortalidade neonatal e avaliação da assistência à gestante e ao recém-nascido. Cad saúde pública [Internet]. 2014 [cited 2015 Nov 13]; 30(Suppl.): S192-207. Acesso em 29/06/2018 Disponível em: http://www.scielo.br/pdf/csp/v30s1/0102-311X-csp-30-s10192.pdf.

13. LEAL MC, PEREIRA APE, DOMINGUES RMSM, et al. Intervenções obstétricas durante o trabalho de parto e parto em mulheres brasileiras de risco habitual. Cad. saúde pública. 2014; 30(supl. 1): 17-31. Acesso em 27/06/2018.

14. MINISTÉRIO DA SAÚDE. Cadernos Humaniza SUS - Volume 4: Humanização do parto e do nascimento. Brasília, DF: UECE/ Ministério da Saúde. 2014. Acesso em 26 de junho, 2018, disponível em http://www.redehumanizasus.net/sites/default/files/ caderno_humanizasus_v4_humanizacao_parto.pdf.

15. NARCHI NZ, CRUZ EF, GONÇALVES R, O papel das obstetrizes e enfermeiras obstetras na promoção da maternidade segura no Brasil. Ciênc Saúde Coletiva [Internet]. 2013; [cited 2017 Mar 17]; 18(4):1059-68. Acesso em 28/06/2018. Disponível em: http://www.scielosp.org/pdf/csc/v18n4/19.pdf.

16. NUNES, DC, SILVA LAM, Humanização na assistência de Enfermagem durante gestação, parto e puerpério e seus desafios na promoção de saúde. Revista Mineira de Ciências da Saúde. 2012. ISSN 2176-2244. Acesso em 28/06/2018. Disponível em: http://www.scielo.br/pdf/ean/v19n1/1414-8145-ean-19-01-0181.pdf.

17. ORGANIZAÇÃO MUNDIAL DA SAÚDE - OMS. Prevenção e eliminação de abusos, desrespeito e maus-tratos durante o parto em instituições de saúde. Genebra, 2014 Autor. Acesso em 30 de junho, 2018, disponível em http://apps.who.int/iris/bitstream/10665/134588/3/WHO_RHR_14.23_por.pdf.

18. PORTARIA n. 1.459, de 24 de junho de 2011. Institui no âmbito do Sistema Único de Saúde - SUS - a Rede Cegonha. Brasília, DF: Ministério da Saúde. 2011. Acesso em 26 de junho, 2018, disponível em: http://bvsms.saude.gov.br/bvs/saudelegis/gm/2011/prt1459_24_06_2011.html.

19. SANFELICE CA, ABBUD FLD, et al. A. Do parto institucionalizado ao parto domiciliar. Revista Rene, 2014 . Vol. 15(2), 362-370. Acesso em 29/06/2018. Disponível em: http://dx.doi.org/10.15253/2175-6783.2014000200022.

20. SILVA AA, SOUZA TGG, MUNHOZ MA, et al. Violência Obstétrica: Perspectiva da Enfermagem. Revista Rede de Cuidados em Saúde. 2015. 32 (3): 479-86. Acesso em 29/06/2018. Disponível em: http://www.scielo.br/pdf/tce/v26n2/pt_0104-0707-tce-26-02-e06500015.pdf.

21. SOUSA A, MM, SOUZA KV, REZENDE EM et al. Práticas na assistência ao parto em maternidades com inserção de enfermeiras obstétricas, em Belo Horizonte, Minas Gerais. Esc Anna Nery [Internet]. 2016; [cited 2017 Mar 16]; 20(2):324-31 Acesso em 28/06/2018. Disponível em: http://www.scielo.br/pdf/ean/v20n2/1414-8145-ean-20-020324.pdf.

22. WOLFF LR, WALDOW VR. Violência consentida: mulheres em trabalho de parto e parto. Saúde \& Sociedade, [Internet]. 2008 [cited 2018 Fev 13]; 2018 17(3), 138- 151. Acesso em 29/06/2018 Disponível em: http://www.scielo.br/scielo.php?script=sci_arttext\&pid=S0104-12902008000300014. 